\title{
Kiek in die Welt von Marga von Etzdorf und Halbschatten von Uwe Timm ${ }^{1}$
}

\section{Teresa Martins de Oliveira}

Universidade do Porto - ILC

Zusammenfassung: Die frühzeitig verstorbene deutsche Flugpionierin Marga von Etzdorf ist die Hauptfigur des 2008 erstveröffentlichten Romans Halbschatten von Uwe Timm. Der Roman ist nicht nur eine Collage aus mehreren Stimmen, Perspektiven und Zeitebenen, sondern auch ein Puzzle aus intertextuellen Bezügen. Als bevorzugter Prätext erweist sich der autobiographische Reisebericht von Marga von Etzdorf Kiek in die Welt. In meinem Beitrag möchte ich auf diesen zu Unrecht vergessenen Text zurückkommen, um den Dialog zwischen beiden Texten und das semantische Surplus, das diese intertextuelle Beziehung dem Roman von Timm verleiht, hervorzuheben.

Schlüsselbegriffe: Uwe Timm, Marga von Etzdorf, deutscher Gegenwartsroman, Reiseliteratur, Erinnerung, Intertextualität

Resumo: A pioneira de aviação alemã Marga von Etzdorf, prematuramente desaparecida, é a figura principal do romance Halbschatten de Uwe Timm (2008). O romance é não só uma colagem de vozes, perspetivas e diferentes planos temporais, mas também um puzzle de referências intertextuais. Como hipotexto privilegiado releva-se o relato de viagem de Marga von Etzdorf Kiek in die Welt. Neste artigo pretendo regressar a este relato, tão injustamente esquecido, para mostrar o diálogo entre ambos os textos e a mais-valia semântica que esta relação intertextual representa para o romance de Timm.

Palavras-chave: Uwe Timm, Marga von Etzdorf, romance alemão contemporâneo, literatura de viagem, memória, intertextualidade 
Die frühzeitig verstorbene deutsche Flugpionierin Marga von Etzdorf ist die Hauptfigur des 2008 erstveröffentlichten Romans Halbschatten von Uwe Timm. ${ }^{2}$ Neben ihrer Biographie werden andere, ebenfalls im Invalidenfriedhof in Berlin begrabene namhafte Personen, meistens Militärs, und deren Lebensgeschichten thematisiert. Der Roman ist also Teil der Erinnerungs- und Gedächtnisliteratur, die seit den 1990ern in Deutschland einen Aufschwung feiert und gehört $\mathrm{zu}$ Uwe Timms historischen Dokumentarromanen. ${ }^{3}$

Den Tendenzen des postmodernen Romans folgend, ist der Roman nicht nur eine Collage aus mehreren Stimmen, Perspektiven und Zeitebenen (es sprechen historische und fiktive Figuren seit der Zeit der Befreiungskriege), sondern auch ein Puzzle aus intertextuellen Bezügen. Als bevorzugter Prätext erweist sich der autobiographische Reisebericht von Marga von Etzdorf Kiek in die Welt, ${ }^{4}$ den Uwe Timm im Nachwort seines Romans auch als solchen erwähnt. In meinem Beitrag möchte ich auf diesen zu Unrecht vergessenen Text zurückkommen, um den Dialog zwischen beiden Texten und das semantische Surplus, das diese intertextuelle Beziehung dem Roman von Timm verleiht, hervorzuheben.

Für meine Analyse bietet sich die Verwendung des Intertextualitätskonzepts von Renate Lachmann an, nicht nur weil sie den Intertextualitätsbegriff mit den Gedächtnisstudien in Verbindung gestellt hat (Lachmann 1990), sondern auch, weil darüber hinaus in ihrem Konzept von Intertextualität die semantischen Mechanismen der Sinneserzeugung besonders wichtig sind. ${ }^{5}$ Für die Bestimmung der Kontaktbeziehungen zwischen den Texten entwickelte sie ein dreidimensionales Modell: Partizipation (d. h. Wiederholung oder Nachahmung des Ausgangstextes); Transformation (Transformierung oder Verbergung des Ausgangstextes) und Tropik (Wiederschreibung und Überbietung des Ausgangstextes), deren Grenzen als fluid $\mathrm{zu}$ betrachten und mit einer bestimmten Sinneserzeugung in Beziehung zu setzen sind. ${ }^{6}(\mathrm{sic})$ Für meine Überlegungen ist ebenfalls wichtig, was Ansgar Nünning über die Verbindung zwischen Gedächtnis und historischen Kontext behauptet, nämlich über die enge Verbindung zwischen Gedächtnisliteratur und den Bedürfnissen der Zeit, in der sie produziert wird (Nünning 2003: 5). 
Beginnen wir mit dem heute vergriffenen und in wenigen Bibliotheken verfügbaren Text von Marga von Etzdorf. Die von mir verwendete Ausgabe, die auch von Uwe Timm erwähnt wird, enthält den von von Etzdorf geschriebenen Reisebericht und ein Nachwort. Wie Timm bemerkt, muss der Text, der als Copyright das Jahr 1931 angibt, später veröffentlicht worden sein, da das Nachwort zwei Nachrufe auf die Flugpionierin, die 1933 gestorben ist, enthält (HS, 268).

Der Reisebericht beginnt mit (knappen) Informationen aus Kindheit und Jugend von Marga von Etzdorf. Als Enkeltochter eines kurz zuvor geadelten preußischen Offiziers, wuchs sie nach dem frühen Tod ihrer Eltern zusammen mit ihrer Schwester bei den Großeltern auf einem Landgut in der Niederlausitz und in Berlin auf. In den drei folgenden Kapiteln werden in vertrautem und unterhaltsamem Ton die verschiedenen Etappen ihrer Ausbildung erläutert, was dem Leser ermöglicht, den Umfang einer Pilotenausbildung in den 1930ern zu verfolgen, von den Pilotenprüfungen für Sport-, Kunst- und Segelflüge bis hin zum B-Schein, der das Fliegen schwerer Flugzeuge erlaubt und den sie als erste deutsche Frau erhielt. Zu erwähnen ist auch ihre Arbeit als Kopilotin bei der Lufthansa erst 60 Jahre später, in den 1990ern, wird bei der deutschen Fluggesellschaft wieder eine Frau im Cockpit sitzen. Es folgte im Jahre 1930 der Kauf eines Junkers A 50 "Junior", den sie auf ihren eigenen Kosenamen, "Kiek in die Welt", von der Pilotin Thea Rasche taufen lie $\beta$. Das im Text oft anthropomorphisierte Flugzeug wird zu einer Art Alter Ego von Marga von Etzdorf:7 Mit ihm nimmt sie als Kunstflugpilotin an Flugtagen teil, transportiert Passagiere und macht Luftwerbung, Arbeiten, die ihr finanzielle Unabhängigkeit ermöglichen. Die Sprache ist klar und informativ, an diversen Stellen auch voller Poetizität, die vorherrschende Perspektive "von oben" wechselt sich mit Beschreibungen während Autofahrten ab. 8

Die folgenden Kapitel widmen sich je einem der von von Etzdorf geflogenen Langstreckenflüge. Der erste Flug führt sie im September 1930 in Begleitung eines Passagiers und eines anderen Flugzeugs nach Konstantinopel. Es ist eine "Bewunderungsreise", in deren Bericht sie nicht nur über praktische und logistische 
Angelegenheiten, sondern auch über touristisch interessante und landschaftlich schöne Gegenden schreibt.

Im darauffolgenden Kapitel wird von Etzdorfs erster alleine vollzogener Langstreckenflug über Deutschland, der Schweiz, Südfrankreich, Spanien und Nordafrika bis Teneriffa beschrieben. Auf dem Rückflug kommt es beim Abflug auf Sizilien zu einem Unfall, und die Maschine muss in das deutsche Junkers-Werk zurückgebracht werden. Dieser Vorfall stellt ihre erste schwerwiegendere "Bruchlandung" dar. Das achte und letzte Kapitel beschreibt die Reise von Berlin nach Tokio, die sie im August 1931 in elf Tagen über Moskau, die Transsibirische Eisenbahnlinie entlang bis zum Baikalsee, dann nach Korea, Hiroshima, Osaka und zuletzt nach Tokio, wo der Reisebericht endet, führt.

Auf den folgenden Seiten werde ich mich auf von Etzdorfs Darstellung während ihres Reiseberichts konzentrieren, insbesondere auf die Beziehung, die die Fliegerin mit Deutschland in der Zeit des aufsteigenden Nationalsozialismus unterhielt.

Am Anfang ihres Textes, in dem autobiographische Inhalte ein wichtiges Element darstellen, beschreibt von Etzdorf in äußerst positiver Art und Weise die Freiheit, die sie auf dem großelterlichen Gut genossen hatte (KW 11-12). Ihre Lebensart, die den Gewohnheiten des damaligen Junkertums entsprach, erinnert den Leser an Beschreibungen der realistischen Erzählungen des 19. Jahrhunderts, was an Nünnings Äußerungen über die literarische Inspiration der autobiographischen Berichte erinnert (Nünning 2008).

Die Schwierigkeiten, die von Etzdorf hatte, eine Ausbildung als Fliegerin abzuschließen und eine Karriere als Pilotin einzuschlagen, werden nicht verschwiegen. Von Etzdorf erwähnt, dass ihr als Frau der Zugang zu den meisten Fliegerschulen und eine Festanstellung bei einer kommerziellen Fluggesellschaft verwehrt blieben (KW 29, 55-56). Nichtsdestotrotz betrachtet sie ihre Arbeit als Pilotin nicht aus einem emanzipatorischen Blickwinkel oder als ein Akt einer feministischen Auflehnung. Sie lässt beispielsweise die Passagiere, die sie fälschlicherweise für einen Mann halten, wenn sie in Fliegerkleidung aus dem Cockpit springt, im Glauben, dass ein Mann das Flugzeug geflogen habe. ${ }^{9}$ Im Großen und Ganzen werden die Geschlechterrollen nicht wirklich thematisiert und unkompliziert und „deserotisiert” dargestellt - so erzählt sie beispielsweise in lustigem Ton, wie sie das 
einzig freie Zimmer mit einem männlichen Passagier oder eine Kaserne mit vielen schlafenden Soldaten teilen muss (KW 65, 152). Es ist anzunehmen, dass dieses unbekümmerte und brüderliche Zusammenleben mit den anderen Flugkameraden den Gewohnheiten unter den Fliegern (KW 34, 50) und auch den neuen Sozialisierungsnormen zwischen den Geschlechtern, vor allem unter den sozialen und kulturellen Eliten und unter den Sportlern dieser Jahre entspricht (KW 127). Aber die „Deserotiesierung” der Beziehungen zwischen Mann und Frau ist auch als eine Anpassung an die Normen des moralischen Verhaltens der damaligen Gesellschaft zu betrachten. Von Etzdorf, die gezwungen wurde, alleine zu reisen, lange Zeit von der Familie getrennt zu verbringen und vor allem alleine unter Männern zu verkehren, was für die damaligen Verhältnisse für eine junge Frau sehr selten war, versucht ein Selbstbild zu entwerfen, das jeglicher erotischer Konnotation entbehrt (in Elly Beinhorns Memoiren ist ein weiteres Bespiel für diese Haltung zu finden). ${ }^{10}$

Was die Frauenfrage betrifft, die zur damaligen Zeit im Zentrum vieler Diskussionen stand, ist zu erwähnen, dass sie in einer gewissen Weise "exportiert" wird, da sie erst in Beziehung auf die Situation der Frau in Spanien explizit behandelt wird. Von Etzd orf betont, wie schlecht die Situation der spanischen Frauen im Vergleich mit der der deutschen ist (KW 103-104, 106, 121). Überhaupt steigt die kritische Bewertung anderer Länder und der paternalistische Ton je weiter Marga von Etzdorf nach Süden kommt und parallel dazu das "Loblied auf Deutschland" ansteigt. Schon bei der ersten Landung in Frankreich beschreibt sie die unsauberen Verhältnisse in einem französischen Haushalt (K 101). ${ }^{11}$

Der nationalistische Ton, den sie ergreift, wenn sie mit Begeisterung behauptet, dass sie für Deutschland fliege, erscheint dem heutigen Leser als sehr fremd. Wie bei vielen Texten von anderen Fliegern dieser Zeit, werden die interkontinentalen Flüge als Akte politischer und wirtschaftlicher Diplomatie begründet.12 Von Etzdorf versteht sich als Botschafterin für Völkerverständigung und Frieden und andererseits auch als Repräsentantin deutscher Wirtschaft und Technologie. Sie freut sich sehr, wenn sie Deutsche oder deutschsprechende Menschen trifft (KW 84, 92, usw.); oder sogar wenn sie einen deutschen Friedhof aus dem ersten Weltkrieg besuchen darf (KW 86); die deutschen 
Konsuln werden überall als außerordentlich gastfreundlich und hilfsbereit beschrieben (KW 123, 165, usw.). Auch spricht sie mit Begeisterung von ihren Besuchen bei den Einwohnern der ehemaligen deutschen Gebiete (KW 64-66, 84) oder Kolonien, denen sie Hoffnung auf ein Wiederaufbau der Heimat vermitteln wollte. ${ }^{13}$ Ähnlich sollte der Effekt auf den Leser sein: Nationaler Stolz und Patriotismus sollten gefördert werden, da sie bestimmt auf finanzielle Unterstützung von Seiten der deutschen Regierung und der deutschen Industriewerken abzielte. ${ }^{14}$

Nicht zu vergessen ist die Tatsache, dass von Etzdorfs Reisebericht, wie bereits erwähnt, erst nach ihrem Tod veröffentlicht wurde, wie das zweiteilige Nachwort bestätigt. Zu diesem Epilog gehören zwei kurze Texte, als "Ausklang” und "Nachwort” betitelt, und die von Heinz Orlovius, Leiter der Pressestelle des Reichluftfahrtministeriums und Autor von verschiedenen Büchern über die Luftfahrt, namentlich über die Luftwaffe (der erste) bzw. von der Fliegerin Katja Heidrich (der zweite) verfasst wurden. Diese Texte haben (unter anderem) die Aufgabe, die zeitliche Lücke zwischen der Landung in Japan und von Etzdorfs Tod zu füllen: Der erste hebt den großen Erfolg von Margas Japanreise sowie ihre politische und diplomatische Bedeutung hervor; der zweite beschreibt die Schwierigkeiten der Rückreise aus Japan, den Absturz in Bangkok, während dem von Etzdorf ihr geliebtes Flugzeug verliert und schwer verletzt wird und betont zudem die Schwierigkeiten der Fliegerin, eine Finanzierung der nächsten Reise zu erhalten. Die ersehnte Reise nach Australien endet kurz nach dem Abflug mit der Bruchlandung in Aleppo, Syrien, und dem Freitod der Fliegerin in einem Raum des Offizierskasinos. "Weshalb? Warum? - Es steht uns nicht zu, darüber zu richten, [...]" (KW 174), liest man in dem gefühlsvollen Kommentar von Katja Heidrich, die die offizielle Version über von Etzdorfs Tod bestätigt: Sie habe befürchtet, keinen neuen Sponsor für weitere Flüge zu finden, was wiederrum das Ende ihrer Flugkarriere bedeutet hätte. Auf einen möglichen Waffenschmuggel, der gegen internationale Flugvorschriften und die Bestimmungen des Versailler Vertrags verstoßen hätte, wurde nicht eingegangen.

Im letzten Paragraph des Nachworts wird Marga von Etzdorf nicht nur als "Pionier besten Formats" und "Kamerad" (beide Substantive in der männlichen Form) bezeichnet, es 
werden ihr auch Charakterzüge zugesprochen, die nicht nur traditionell als männlich, sondern als typisch für einen (wilhelminischen) Offizier betrachtet werden: "Pflichtgefühl, Verantwortungsbewusstsein, Bescheidenheit und Edelmut" (KW 175). Dazu kommt, dass das Ausbleiben einer Antwort auf die selbstformulierte Frage ("Weshalb? Warum?") der verschwiegenen Erklärung einen erhabenen Sinn verschafft, der von Etzdorfs Eintritt in das Heldenpantheon vorbereitet. Zur Sakralisierung der Heldin tragen ihre Bestattung auf dem Invalidenfriedhof und die Inschrift auf ihrem Grabstein bei - eine Zeichnung ihres Grabs erscheint am Schluss des Bandes. Darauf ist zu lesen: "Der Flug ist das Leben wert" (KW 175). Dieser Satz, der für immer mit Marga von Etzdorf verbunden bleibt, erhält auf ihrem Grab eine ähnliche Funktion wie die Veröffentlichung ihrer Memoiren. Indem man ihren Tod als das Opfer für einen höheren Wert darstellt, zielt man auf die Rechtfertigung des Freitods. Von Etzdorf ist es nun erlaubt, in die Gruppe der früh verstorbenen Helden einzutreten, die der Nationalsozialismus für sich beanspruchte. Die Fotos von von Etzdorfs feierlichen Aufbahrung zeigen ein typisches Beispiel für die Art der Inszenierung, die der Nazi-Staat nutzte, um junge frühverstorbene Männer und Frauen als Personifikationen des Nazi-Helden darzustellen. Die Veröffentlichung ihres Reiseberichts mit den beiden Epilogen hatte vermutlich eine ähnliche Funktion.

Wenden wir uns nun dem Roman von Uwe Timm zu. Der Handlung des Romans ist nicht leicht nachzugehen: Ein Ich-Erzähler, der gleichzeitig als Adressat und als Alter Ego des realen Autors fungiert, besucht, zusammen mit einem Stadtführer, den Invalidenfriedhof in Berlin. Dieser Friedhof, auf dem "die deutsche, die preußische Geschichte begraben [liegt]" (HS 9), war bis 1989 militärisches Sperrgebiet, da die BerlinerMauer dort verlief - er evoziert also vielfache Erlebnisse der deutschen Geschichte. ${ }^{15}$ Während dieser Führung erheben sich nacheinander Stimmen aus den Gräbern, die eigene oder fremde Erlebnisse erzählen oder kommentieren. Unter ihnen hört man die Stimmen des preußischen Generals und Heeresreformers von Scharnhorst, des mit der "Endlösung der Judenfrage" beauftragte Nazi Reinhard Heydrich und vieler Flieger, wie Richthofen oder Udet, und auch Stimmen von fiktiven Figuren. Marga von Etzdorf ist in diesem sonderbaren Choral ebenfalls Erzählerin und ein privilegiertes Thema und ihre Geschichte erscheint wie 
in einem Mosaik, mit den Geschichten dieser anderen Figuren verwoben (Uwe Timm nennt den Text ein Oratorium (Bartels 2008).16 So wird die Geschichte der Fliegerin nacherzählt, wobei historisch verbürgte Erlebnisse neben fiktionalen stehen. Unter diesen fiktionalen Elementen ist die Figur von Dahlems hervorzuheben, der eine zentrale Rolle im Roman spielt. Bei ihrer Ankunft in Hiroshima kann von Etzdorf keine Unterkunft finden und der charmante deutsche Konsul (der im Ersten Weltkrieg Jagdflieger war) bietet ihr sein Zimmer an, in dem beide, von einem Vorhang getrennt (darauf deutet der Titel des Romans hin), eine unvergessliche Nacht verbringen. Die ganze Nacht führen sie ein immer intimer werdendes Gespräch, und ab diesem Erlebnis fühlt sich Marga von Etzdorf dem Mann innigst verbunden. Dieser schätzt sie zwar sehr, aber seine Liebe gehört einer Anderen, was er auch Marga anvertraut. Die platonische Beziehung, voller Fort- und Rückschritte, die sich zwischen beiden entwickelt, steht im Zentrum der „Marga-Handlung“. Es ist von Dahlem, der ihr zwei Jahre später vorschlägt, die finanzielle Unterstützung der Nationalsozialisten für Waffenschmuggel und Fotospionage in Syrien anzunehmen.

Bevor ich mich der Analyse der intertextuellen Bezüge zwischen Roman und Reisebericht widme, möchte ich daran erinnern, dass es bei HS, wie bei anderen Texten von Uwe Timm, eine reiche Intertextualität festzustellen ist: Es sind viele Verweise auf andere Texte des Autors sowie anderer Herkunft (Wiese erwähnt die Beziehungen zu den Romanen Am Beispiel meines Bruders und Rot und zur Legende Der Mann auf dem Hochrad (Wiese 2016: 89-91)). Auch Zitate aus Gedichten, Romanen, Briefen, Gebeten und aus der Bibel sowie aus amtlichen Dokumenten sind festzustellen. ${ }^{17}$ Darüber hinaus sind Verweise auf Fotos (HS 220, 224), Filme (HS 10-11) und Interviews (HS 11-12) für die Konstruktion der Hauptfigur wichtig.

Unter den vom Autor benutzten Büchern hebt er in der Nachschrift, wie bereits erwähnt, von Etzdorfs Reisebericht als privilegierten Prätext hervor (HS 268). Trotzdem ist festzustellen, dass die Zitate und Motive aus von Etzdorfs Buch als solche nicht leicht zu entdecken sind. Die Nahtstellen der übernommenen Textteile sind verborgen, es gibt im Roman weder Verweise auf den Prätext noch sind die Textstellen graphisch markiert. Wenn wir auf Renate Lachmanns Modell zurückkommen, so scheint Timms Text sich zwischen 
verschiedenen Polen zu bewegen: der Partizipation, der Transformation und der Tropik, mit einer klaren Tendenz zur ersten, die "im Wiederholen und Erinnern der vergangenen Texte ein Konzept ihrer Nachahmung einschließt" (Lachmann 1990: 39).

Auch aus einer zeitlichen Perspektive betrachtet, scheint HS mit KW eine Beziehung der Kontiguität zu hegen: Man kann sagen, dass die Haupthandlung des Romans da anfängt, wo die Erzählung von von Etzdorf in dem Reisebericht aufhört, als ob sie den Zeitsprung zwischen Margas glücklichen Ankunft in Tokio und ihrem Freitod füllen wolle, um eine mögliche Erklärung für den unerwarteten Schluss zu finden.

Was die andere Hauptfigur, die fiktionale Figur von Dahlems, und die Konturen der "ersten Nacht" angeht, so entstammen sie der Phantasie des Autors, können aber mit dem Erlebnis mit dem deutschen Konsul in Hiroshima, wie er von Marga im Reisebericht beschrieben wird, in Verbindung gestellt werden:

Aber der Rettungsengel war schon unterwegs. Es war der deutsche Konsul, der meinetwegen aus Kolbe kam, ohne da $\beta$ (sic) ich es ahnte. In der Nacht bewohnte er das Zimmer neben meinem, und am Morgen stellte er sich durch die Papierwand vor. Da der Konsul zu allem Glück noch ein alter Kriegsflieger war, ging alles am Morgen glatt und schnell, [...]. (KW 168)

Die intertextuelle Präsenz zeigt sich auf diskursiver Ebene bei den vielen Echos, Paraphrasen und Zitaten. Es sind vor allem in den Beschreibungen der Reisen, die Marga von Dahlem erzählt, dass man lange Zitate aus KW findet. ${ }^{18}$ Neben genauen Übernahmen vom Prätext, sind Zusammenfassungen und Paraphrasen zu finden, manchmal wird der Text an einen mündlichen Ton angepasst oder von Kommentaren der Protagonisten oder anderer Figuren unterbrochen, die auf alternative Interpretationen hinweisen, und man kann mit Sicherheit behaupten, dass diese zitierten Textstellen $\mathrm{zu}$ den poetischsten von Timms Roman zählen. Obwohl diese intertextuellen Bezüge vor allem bei den Reden von Marga zu finden sind, wird der Text von KW und sogar von den beiden Nachworten auch in der Rede anderer Figuren übernommen. Auch viele Erlebnisse und Informationen aus KW werden in HS wiederholt. ${ }^{19}$

Vergleicht man die Konturen von Margas Figur und ihre Geschichte in beiden 
Texten, werden die von Uwe Timm gewählten Akzentverschiebungen deutlich. Die erste (und vielleicht wichtigste) Verschiebung ist die Annäherung der Figur Marga von Etzdorf und ihrer Lebensgeschichte an heutige Zustände, Sehnsüchte, Empfindungs- und Denkweisen zu nennen. Wichtig ist ebenfalls, dass man sie mit sozioökonomischen, politischen, identitären und philosophischen Aspekten in Verbindung stellt. Informationen über die soziale Herkunft von von Etzdorf werden (fast) vergessen. ${ }^{20}$ Finanzielle Schwierigkeiten, die im Reisebericht zwar erwähnt werden, aber vermutlich entsprechend den gesellschaftlichen Normen ihrer Zeit und Herkunft kein Thema darstellten, stehen jetzt im Vordergrund. ${ }^{21}$ In KW erzählt Marga zwar von den Schwierigkeiten, Sponsoren für die Langstreckenflüge zu finden (KW 143),,22 aber die Teilnahme an Kunstflugwettbewerben und der Passagiertransport werden wiederholt als angenehme Erfahrungen beschrieben, während in HS diese Episoden, wie auch die vielen Vorträge, nur als lästige Arbeit angesehen werden, um an Geld zu kommen. ${ }^{23}$ Auch die Bezüge hinsichtlich der Unterstützung, die sie von den deutschen Flugzeugwerken und von internationalen Unternehmen erhielt, und die sie - eine regelrechte Marketingstrategie verfolgend - in dem Reisebericht wiederholt erwähnt, ${ }^{24}$ verwandeln sich in HS zu klaren Denunzierungen der ökonomischen Interessen der multinationalen Konzerne.

Was die Rolle der Frau betrifft, ist festzuhalten, dass die Diskriminierung der Fliegerinnen, die im autobiographischen Text nur andeutungsweise erwähnt wird, in HS auch nicht ausführlicher thematisiert wird. Auch hier wird die Episode von Belchite (Spanien) hervorgehoben, sie wird sogar detaillierter erläutert in einem Ton zwischen heiterem Scherz und arroganter Ironie, der auch im Reisebericht zu finden ist. 25

Bis jetzt wurde konstatiert, dass Uwe Timm Margas Charakterzüge im Großen und Ganzen so übernimmt, wie sie in KW vorgestellt werden, doch bei Margas sexueller Orientierung und bei der Beziehung zu anderen Fliegerinnen orientiert er sich eher an aktuellen Gesellschaftsnormen. Das Fehlen von Anspielungen auf Liebesbeziehungen oder erotische Abenteuer bei KW und die schon erwähnte „deserotisierte“ Beziehung zwischen den Geschlechtern wird in HS von einer erweiterten Diskussion über Margas sexuelle Orientierung ersetzt, an der verschiedene Figuren teilnehmen (HS 31, 156, 215). Margas 
Liebe für von Dahlem kann zwar als eine mögliche Antwort auf diese Frage verstanden werden, aber andererseits erzählt sie selbst von der Beziehung zu einer "Freundin" (HS 205-209), was das Motiv der sexuellen Unbestimmthei zurückbringt.

Auch bei den Beziehungen, die Marga mit anderen Fliegerinnen unterhält, taucht bei HS ein Motiv auf, das unsere heutige Gesellschaft bewegt. So wird der Sportgeist und die gesunde Kameradschaft unter den Fliegern, die in KW wiederholt erwähnt werden und die vermutlich auch eine Euphorisierung der tatsächlichen Beziehungen darstellen (andere autobiographische Texte aus den 1930er Jahren thematisieren, wie Flieger und Fliegerinnen unter sich wetteifern), ${ }^{26}$ durch Konkurrenz und Wettstreit zwischen den Fliegern ersetzt. In HS ist das Wetteifern zwischen Marga von Etzdorf und der Engländerin Amy Johnson ein Leitmotiv. ${ }^{27}$

Dass die unterschiedlichen Gattungen zu denen die analysierten Texte gehören dazu beitragen, verschiedene Schwerpunkte bei der Darstellung von Margas Geschichte zu setzen, ist leicht verständlich. So ist zum Beispiel, Marga von Etzdorf als Hauptfigur des Romans facettenreicher und widerspruchsvoller als im Reisebericht, was durch die verschiedenen Stimmen und Perspektiven und durch die vielfältigen Interpretationsmöglichkeiten erreicht wird. Erwähnt seien ebenfalls die neuen poetischen Ebenen, die die vielen in den Roman neu eingeführten Motivketten ermöglichen. Einige dieser Motive (wie der Schatten, die Wolken und der Gesang) sind Träger eines symbolischen Wertes, den man im Reisebericht trotz seiner an vielen Textstellen nachweisbaren poetischen Sprache nicht findet. Andere poetische Motive werden vom Prätext übernommen oder erweitert oder bekommen durch Montage und Assoziation zu neuen Textpassagen neue und subtile Bedeutungen.28

Als Beispiel hierfür ist die Episode vom Vortragen des Heinegedichts in der Wüste zu erwähnen, die schon bei KW gefühlvoll erzählt wird. In HS kommen nicht nur neue romantische Motive hinzu (die intimere Gruppe um Marga, das Lorca-Gedicht, seine Thematik und die geschilderten Sinneseindrücke sowie das Zitat aus dem Heinegedicht), ${ }^{29}$ sondern es wird eine politische Ebene neu konstruiert. Die Einführung von dem Lorca- 
Gedicht macht auf die politische Dimension von Heines Dichtung aufmerksam. Auch Heines jüdische Abstammung wird mehrmals erwähnt und ebenfalls funktionalisiert.

Vielleicht wäre sie auch Testpilotin der Luftwaffe geworden wie Hanna Reitsch.

Die Etzdorf nicht, sagt der Graue. Wer Heine-Gedichte auswendig kann, der nimmt einen anderen Weg.

Wer weiß?

Ja, wer weiß. (HS 251)

Bezüglich der Übernahme von Motiven aus KW ist auch das Motiv des Fliegens zu nennen. In KW werden konkrete Informationen über den Erziehungsweg der Pilotin und auch besondere Erlebnisse am Steuer ihres Flugzeugs, mit Hervorhebung von technischen und logistischen Bedingungen neben Erzählungen aus speziellen Reisen und Beschreibungen von Luftlandschaften, betont. Abwechselnd werden Margas Begeisterung und Angsterlebnisse dargestellt, die Sprache ist lebendig und oft poetisch. Es ist festzuhalten, dass bei KW das Fliegen für Marga ein Treffen mit sich selbst und mit den Elementen darstellt.

In HS taucht das Motiv des Fliegens mit metaphorischer, poetischer oder philosophisch-literarischer Bedeutung, aber auch mit einer charakterisierenden Funktion wiederholt auf. Charakterzüge wie Optimismus und Lebenslust, die Marga in KW zeigt, werden in HS mit dem Fliegen konnotiert: Das Singen beim Fliegen wird als Leitmotiv erwähnt (HS 11, 69, 136); Marga redet in einem Interview von der "Schwerelosigkeit" beim Fliegen (HS 11), Miller spricht vom Fliegen als Poesie. ${ }^{30}$ Extreme Erfahrungen werden vorgezogen und deshalb werden bei den Langstreckenflügen mehr die Schwierigkeiten als die glücklichen Erlebnisse beschrieben. Wunsch nach der Ferne und der Schwerelosigkeit (HS 11) als Ausdruck der Todessehnsucht (HS 146), aber auch als Überwindung von menschlicher Begrenztheit durch Zeit, Raum und Schwerkraft, und von den Begrenztheiten als Frau, Fliegen als Ausdruck des Fortschritts, als Vollzug von Gottes Willen, der Mensch solle sich die Erde untertan machen, wie Marga in ihrem Vortrag erwähnt, der mit dem lapidaren Satz abschließt: "Der Flug ist das Leben wert” (HS 174-176). Mit dem Motiv des 
Flugs lässt sich ebenfalls die Figur des Engels in Beziehung setzen. ${ }^{31}$ Mit diesem teilt Marga das Fliegen und die Schwerelosigkeit, die Androgynie (HS 18), das Los der Botin (HS 123126) und das tragische Schicksal des Sturzes, Marga wird zur „Pechmarie“ (HS 221) und erleidet dadurch das tragische Los des gefallenen Engels (HS 204 und 219).

Zuletzt betrachte ich die politische Bedeutung des Fliegens, die im Roman ein zentraler Wert zugestanden wird. Margas nationalistischer Diskurs in dem Reisebericht und die politische Instrumentalisierung der Fliegerin durch die Nazis, die wir bei KW feststellten, werden in HS nicht nur direkt thematisiert, sondern von verschiedenen Stimmen diskutiert. Dass es Nazi-Figuren sind, die Margas Verbindung zum Regime bestätigen, trägt dazu bei, Marga als NS-Opfer und nicht als NS-Sympathisantin darzustellen. Auch der explizite Vergleich mit anderen Fliegerinnen, wie z.B. Hanna Reitsch, trägt dazu bei, Marga von Etzdorf von dem Verdacht der Kooperation mit den Nazis zu befreien. Es wird klar, dass ihr frühzeitiger Tod noch in einer Anfangsfase des Naziregimes erfolgte, und dass man von keiner bewussten Kolaboration sprechen kann.

Wie es typisch für den Gegenwartsroman ist, werden endgültige Antworten vermieden. Es wird aber nie in Frage gestellt, dass Marga sich erst in einem Zustand großer innerer Zerbrechlichkeit entschieden hat, den Auftrag des Waffenschmuggels und der Spionage zu übernehmen. Diskutiert (oder suggeriert) werden stattdessen die Gründe die zu ihrem Freitod geführt haben könnten. Die Verzweiflung über den eigenen Fehler beim Landen, der ihren Ruf als Fliegerin endgültig beeinträchtigten würde; die Todessehnsucht, die sie schon bei der Liebe zum Fliegen zeigte, die große Liebesenttäuschung; der Schmerz und die Reue über die eigene Fehlentscheidung; der Wunsch, sich die eigene Würde zu bewahren:

Es war Stolz. Würde. Etwas, was mehr und mehr verloren geht. Ein Beispiel geben. Beispielhaft für sich selbst, zugleich auch für andere. Etwas, was uns empört, etwas, was wir nur mit dem Letzten, den Äußersten deutlich machen können. Was jeden Kleinsinn in Frage stellt. Die radikale Freiheit in Anspruch nimmt. Ich zu sein. Die Größe in der Selbstbestimmung. (HS 245)

Die Denunzierung des Nationalsozialismus wird dabei besonders hervorgehoben. Sie 
entspricht ebenfalls dem globalen Sinn des Romans, der, trotz der vielen Verzerrungen durch Vielstimmigkeit und "Halbschatten", aus den vielen fiktionalen und geschichtlichen Erlebnissen klar hervorsticht.

Zuletzt sei noch kurz auf Ansgar Nünnings Überlegung eingegangen, laut der die Erinnerung (und die Erinnerungsliteratur) von den Bedürfnissen des jeweiligen Produktionskontextes weitgehend abhängt. Schon die Tatsache, dass von Etzdorfs Reisebericht postum und im Jahre der Machtergreifung veröffentlicht (oder wiederveröffentlicht?) wurde, deutet auf ein Akt der Propaganda des NS-Regimes hin, für dessen Wirksamkeit die beiden Epiloge, wie gesehen, auf subtile Weise sorgen.

Dagegen hat Uwe Timms Roman den Verdienst, die historische Figur von Marga von Etzdorf vor dem Vergessen $\mathrm{zu}$ retten, und ihre Vereinnahmung durch die Nationalsozialisten zu thematisieren, was letztendlich dazu führt, sie von diesem Ruf zu befreien. Darüber hinaus ruft er die Aufmerksamkeit auf einen mit Unrecht vergessenen Text, der sicherlich eine Wiederveröffentlichung verdient hätte. Aus meiner Analyse wurde deutlich, dass diese semantische Erweiterung mit den Worten Lachmanns mehr im Sinne einer Partizipation als einer Transformation geschieht: Elemente wie Verbergung des früheren Textes, die Tendenz “(...) ihn unkenntlich zu machen und den Text als eigenen zu präsentieren" (Lachmann 1997: 681) deuten zwar auf eine Wiederschreibung im Sinne einer Transformation hin, aber, wie festgestellt wurde, tendieren die übernommenen Erlebnisse und Motive dazu, im Reisebericht schon dargestellte Charakterzüge und Motive genauer zu untersuchen und/oder in ihren möglichen Bedeutungen zu erweitern.

Die intertextuelle Präsenz von Marga von Etzdorfs Reisebericht kann man also aus einer doppelten Perspektive betrachten. Einerseits zählt sie, zusammen mit der Vielstimmigkeit, der Multiperspektivität und den verschiedenen Handlungssträngen zur Offenheit des Textes und zu Timms Metareflexion über die Grenzen einer objektiven Geschichtsschreibung und ihrer literarischen Behandlung. Andererseits aber ist diese intertextuelle Präsenz der Beweis von Timms Wertschätzung seiner Figur, für die er nicht nur die Aufmerksamkeit sondern auch die Sympathie der Leser gewinnen möchte. 


\section{Bibliographie}

Bahners, Patrick (2009), „Seufzen und Sausen“, Frankfurter Allgemeine Zeitung, http://www.faz.net/aktuell/feuilleton/buecher/rezensionen/belletristik/uwe-timmhalbschatten-seufzen-und-sausen-1831626.html, erschienen 06. 07. 2009 (Aufruf 27.12 2015).

Bartels, Gerrit (2008) „Erlösung über den Wolken. Die Fliegerin, der Invalidenfriedhof und die Liebe: ein Besuch bei dem Schriftsteller Uwe Timm“, Der Tagesspiegel, http://www.tagesspiegel.de/kultur/literatur/literatur-erloesung-ueber-denwolken/1316364.html, erschienen 04.09.2008 (Aufruf 27.12. 2015).

Bernsmeier, Helmut (2014), Uwe Timm. Halbschatten, Stuttgart, Reclam.

Etzdorf, Marga von (1931), Kiek in die Welt. Als deutsche Fliegerin über drei Erdteile, Berlin, Union deutsche Verlagsgesellschaft.

Jandl, Paul (2008), "Uwe Timms ambitionierter Deutschlandroman «Halbschatten». Zwischen Himmel und Hölle", Neue Zürcher Zeitung, http://www.nzz.ch/zwischen-himmelund-hoelle-1.839581,erschienen 17.09. 2008 (Aufruf 27. 12. 2015).

Lachmann, Renate (1990), Gedächtnis und Literatur. Intertextualität in der russischen Moderne, Frankfurt/Main, Suhrkamp, 1990.

-- /Schamma Schahadat (1997), „Intertextualität“ in: Helmut Brackert/ Jörn Stückrath (Hrsg.) Literaturwissenschaft: Ein Grundkurs, Reinbek bei Hamburg, Rowohlt, 677-686.

Nünning, Ansgar (2003), "Editorial: New Directions in the Study of Individual and Cultural Memory in Memorial Cultures, in: A.N. (ed.), Journal for the Study of British Cultures. Fictions of Memory, vol. 10, 1, 3-9.

-- (2008),"Zur mehrfachen Präfiguration/Prämediation der Wirklichkeitsdarstellung im Reisebericht: Grundzüge einer narratologischen Theorie, Typologie und Poetik der Reiseliteratur" in : Marion Gymnich/ Ansgar Nünning/ Vera Nünning u.a. (Hrsg.), Points of 
Arrival: Travels in Time, Space, and Self / Zielpunkte: Unterwegs in Zeit, Raum und Selbst, Tübingen, Francke, 11-32.

Timm, Uwe (2015), Halbschatten, München, Deutscher Taschenbuchverlag [2005].

Wiese, Astrid (2016), Uwe Timm. Halbschatten, Stuttgart, Klett.

Zegenhagen, Evelyn (2007), »Schneidige deutsche Mädel«. Fliegerinnen zwischen 1918 und 1945, Göttingen, Wallenstein.

Teresa Martins de Oliveira ist Professorin für deutsche Literatur und Literaturwissenschaft an der Geisteswissenschaftlichen Fakultät der Universität Porto, Portugal. Forschungsschwerpunkte: vergleichende Literatur, Genderforschung; deutschsprachige (Frauen-)Literatur der Gegenwart; Judentum und weibliche Identität in der deutschsprachigen Literatur des 19. und 20. Jahrhunderts; Publikationen zu Fontane, Kafka, Elsa Bernstein, Annemarie Schwarzenbach, Max Frisch, Peter Handke, u.a. 


\section{Fussnoten}

${ }^{1}$ Die Arbeit ist Teil des Forschungsprojekts "Interidentidades/Interculturalidades" des Instituto de Literatura Comparada Margarida Losa da Faculdade de Letras da Universidade do Porto und wird durch die Fundação para a Ciência e a Tecnologia im Rahmen des Projekts «UID/ELT/00500/2013 | POCI-01-0145-FEDER007339» gefördert.

2 Timm, Uwe (2015), Halbschatten, München, Deutscher Taschenbuchverlag [2005]. Im Text wird der Roman mit HS und der entsprechenden Seitenzahl bezeichnet.

${ }^{3}$ In seinen historischen Dokumentarromanen bzw. in seinen Romanen autobiographischer Prägung behandelt Timm wichtige Themen der deutschen Geschichte des 20. Jahrhunderts, wie der zweite Weltkrieg und die Schuldfrage (z.B. Am Beispiel meines Bruders (2003)) und die 60er Jahre in der BRD (Heißer Sommer (1974); Rot (2001); Der Freund und der Fremde [2005)). In HS wird die deutsche Geschichte des 19. und des 20. Jahrhunderts thematisiert, mit besonderer Hervorhebung der Zwanziger Jahre und der Zeit des Nationalsozialismus.

${ }^{4}$ Etzdorf, Marga von (1931), Kiek in die Welt. Als deutsche Fliegerin über drei Erdteile, Berlin, Union deutsche Verlagsgesellschaft. Im Text wird der Reisebericht mit KW und der entsprechenden Seitenzahl bezeichnet.

5 Über die Interaktion zwischen den Texten schreibt Lachmann: “ Bei Verfahren wie Einlagerung von fremden Texten oder Textelementen in den aktuellen Text [...] geht es ja weder um Beschwörung einer heilen Welt literarischer Tradition noch um den Nachweis untilgbarer Bildung, die als Zitat in den Text versenkt wird, sondern um die semantische Explosion, die in der Berührung der Texte geschieht, um die Erzeugung einer ästhetischen und semantischen Differenz" (ebd.: 57).

${ }^{6}$ Ebd.: 39. Zu diesen Begriffen s., ebenfalls, Lachmann, 1997: 677- 686.

${ }^{7}$ Als Beispiel für diese Anthropomorphisierung s. "Aber auch manchen Sturm haben wir zusammen erlebt, mein "Kiek in die Welt" und ich, und manche böse Stunde haben wir überstanden, wenn uns die Böen packten und herumwarfen, wenn die Wolken die Erde verdeckten, wenn der Wind uns entgegenfegte, da $\beta$ (sic) wir kaum vorwärtskamen" (KW 60). Weitere Beispiele: KW 86, 144, 158-160, 164.

${ }^{8}$ Als Beispiel dient die Textpassage über die Autofahrt durch Prag: "Als er [der Vertreter der Firma Shell] hörte, daß (sic) wir Prag noch nicht kannten, machte er, während die Monteure "Kiek in die Welt" tankten, schnell eine herrliche Autofahrt mit uns durch die wundervolle Stadt" (KW 69). Weitere Beispiele dienen die Autofahrten in der Gegend von Württemberg (KW 40,46); oder die vielen Ausflüge zwischen Prag und Wien (KW 69, 71-74). 
9 "Nach der Landung kamen sie [die Passagiere], um sich bei den "Herren Piloten" für den herrlichen Genu $\beta$ (sic) zu bedanken, und ich habe mich immer gehütet, ihnen diese Illusion durch ein Verraten meiner Stimme zu rauben. Mit einer stummen und männlichen Verbeugung steckte ich alle Komplimente ein" (KW 30). S., ebenfalls, KW 53.

10 S., beispielsweise, Beinhorns autobiographischen Bericht Ein Mädchen fliegt um die Welt (1932).

${ }^{11}$ S., ebenfalls, Kommentare von Marga von Etzdorf über die Verhältnisse in Rumänien (KW 89-90), Barcelona (KW 103) und Belchite (KW 108).

12 Evelyn Zegenhagens schreibt über die metapolitische Aufwertung der Luftfahrt in Deutschland der Zwanziger Jahre: "In der Luftfahrt verband sich das Potential einer symbolischen und realen Überwindung geographischer, technologischer und politischer Begrenzungen und Beschränkungen mit der Hoffnung, aus den Niederungen eines bedrückten individuellen und kollektiven Daseins in eine lichte Zukunft emporzusteigen - ein Identifikationsangebot das nahezu allen politischen Richtungen in der Weimarer Republik Sinngehalte anbot"( Zegenhagen 2007: 275).

13 Darüber hinaus hat Deutschland, laut Zegenhagen, verstanden, dass Frauen mehr als Männer für diese friedliche Botschaft geeignet waren (ebd.).

14 Von Etzdorf nutzt jede Gelegenheit aus um zu erklären, dass sie die Auszeichnungen, die ihr verliehen wurden, als Auszeichnungen an Deutschland betrachtet. Als Beispiel hierfür ist die Ankunft in Teneriffa zu erwähnen: "Auch hier, wie in las Palmas, empfand ich mit besonderer Freude, dass die Begeisterung nicht nur mir, sondern darüber hinaus Deutschland und der deutschen Maschine galt. [...]" (KW 124).

15 Man denkt dabei an die Begriffe „Erinnerungsort“ von Pierre Nora (Les Lieux de Mémoire, 1984-1992) und „Terrain vague“ (2002) von Solà-Morales.

${ }^{16}$ Es ist nicht eindeutig, warum Uwe Timm gerade die Figur von Marga von Etzdorf im Zentrum seiner Erzählung stellt. Bei Gerrit Bartels liest man: „Schon kurz nach dem Mauerfall besuchte Timm den damals ziemlich verwahrlosten Invalidenfriedhof, las im Anschluss ein Buch über die hier Bestatteten und erfuhr dabei, dass auch eine Frau darunter ist: (...)“ (apud Bernsmeier, 74). Betrachtet man den Roman, wird es klar, dass die Figur von von Etzdorf und ihre Geschichte, die von Liebe und Begeisterung geprägt sind, als Gegenpol zu den vielen Gewalterlebnissen, die die meisten der erzählten Geschichten charakterisieren, fungieren. Darüber hinaus zeigt die Geschichte ihres ungeklärten Todes, wie schwierig es ist, über Geschichte zu urteilen. Von Etzdorfs Los passt zu Timms narrative Methode, wie er sie in seinen Poetikvorlesungen beschreibt: „(...) Eine ästhetische Konstruktion, die nicht sagt, so war es, sondern so könnte es gewesen sein, die, indem sie über die Leerstellen zwischen den Textblöcken auf das Fragmentarische des Erfahrbaren, Erinnerbaren verweist, nicht den Anspruch auf das ,Ganze' erhebt“" (apud Bernsmeier 2014: 64). 
17 Eine schnelle Information zu den verschiedenen Verweisen bietet das Kapitel "Wort- und Sacherläuterung" aus Uwe Timm. Halbschatten. Reclam Lektüreschlüssel (Bernsmeier 2014: 42-60).

${ }^{18}$ Die wichtigsten Zitate beziehen sich auf die Flüge nach Wien (KW 62-64, HS 70-72); nach Teneriffa über Spanien (KW 104-109; KW 41-42, 43-46); Cabo Juby (KW 116-11,127, HS 122-123; 129-130) und auf den Rückflug nach Catania (KW 133-141, HS 136, 138-140, 145) und den Flug nach Japan (KW161- 163, 167-168, HS 13-16).

${ }^{19}$ Als Diskrepanz zwischen den Informationen aus beiden Texten, siehe man die Kommentare über Margas Liebe zum Schwimmen in HS (HS 187, 210) und Margas Kommentar aus dem Reisebericht, dass sie nicht schwimmen konnte (KW 118).

${ }^{20}$ Erst im letzten Teil des Romans wird durch die Stimme einer Großtante eine direkte Charakterisierung von Marga gegeben. Dabei wird ihre Situation als Vollwaise, das frühe Interesse für Motoren und Fliegen und ihre sportliche Verfassung hervorgehoben. "Hatte einen alles übersteigenden Willen, zäh, nicht von dem abzubringen, was sie sich in den Kopf gesetzt hatte. Und ein fröhliches Kind [...] Kletterte auf Bäume, schwamm im Teich, (...) (KW 187).

${ }^{21}$ Die Bescheidenheit von Margas Lebensverhältnisse wird im Roman in den kleinsten Dingen akzentuiert. Als Beispiel vergleiche man die Wohnbedingungen in Cabo Juby. "Ein reizendes Appartement mit Schlafzimmer, Wohnzimmer und Bad, mit Aussicht auf den Ozean” (KW 116) transformiert Uwe Timm in „(...) ein Zimmer mit einem kleinen Bad. Das Fenster ging hinaus auf das Meer (HS 122).

22 In KW wird die finanzielle Belastung, die der Erwerb des Flugzeugs bedeutete, verschwiegen (KW 56-58). Dagegen wird in HS sehr detailliert erzählt, wie sie ihr ganzes Vermögen in das Flugzeug investierte und darüber hinaus noch Schulden machen musste (HS 68).

${ }^{23}$ Unter diesem Aspekt ist der Vergleich der Beschreibungen des Flugs von Marga mit einem Passagier nach Wien besonders interessant (KW 60-66, HS 70-72).

${ }^{24}$ Als Beispiel s. Hinweise auf die Unterstützung durch die Firma Shell (z.B., KW 98, 105, 120, 130).

25 Zwar kann man Millers ironische Kommentar zu dem Waschlappen als ein typisch deutsches Toilettenartikel als Demaskierung von Margas Denken betrachten (HS 46), aber die genaueren Angaben zu dem Film, den die Menschen aus Belchite ihr vorführen möchten und den sie schon vor drei Jahren in Berlin gesehen hatte, sowie der Mann, der "in einem schwarzen Samtjackett" außer Atem angelaufen kommt, um ihr den Trauermarsch von Liszt vorzuspielen oder das eben eröffnete öffentliche WC als Sehenswürdigkeit der Stadt (HS 45) scheinen Margas Meinung zu bestätigen und an die Empathie des Lesers zu appellieren.

${ }^{26}$ Als Beispiel lese man Thea Rasche, “...und über uns die Fliegerei”, Berlin, Schützen-Verlag, 1940. 
27 Der fast simultane Japanflug von Marga von Etzdorf und von der Engländerin Amy Johnson und die Verwechslung von beiden Fliegerinnen bei der Ankunft in Japan wird in beiden Texten wiederholt erwähnt. Darüber schreibt Marga in KW:

Eines Tages las ich, dass die durch einen hervorragenden England-Australien-Flug im letzten Jahr bekannt gewordene englische Fliegerin Amy Johnson gleichfalls einen Japan-Flug vorhatte. [...] Als ich aber hörte, dass sich Amy Johnson entschlossen hatte, nicht allein, sondern in Begleitung ihres Fluglehrers zu fliegen, da brauchte ich ihren Flug glücklicherweise nicht mehr als Konkurrenzflug zu betrachten. [...] Aber ich habe das Empfinden, dass durch ihren Flug mein für die deutsche Sportfliegerei erzielte Erfolg in keiner Weise beeinträchtigt, ja eher vielleicht durch ihn der Boden für eine günstige Aufnahme und eine objektive Wertung beider Unternehmen vorbereitet worden ist. (KW 145)

Dagegen reagiert Marga in HS mit großer Besorgnis auf die Ankündigung dieses Fluges und mit großer Betrübnis auf die Verwechslungen in Japan (HS 12-16). Darüber hinaus wird die Bedrohung durch den Johnson-Flug als Empathieerzeuger zwischen Marga und von Dahlem funktionalisiert. Es ist der deutsche Konsul der ihr bei der Ankunft in Hiroshima mitteilt, dass sie die erste Frau ist, "die allein von Europa nach Japan geflogen ist", denn Amy war "nicht allein, sondern mit ihrem Fluglehrer geflogen" (HS 16).

${ }^{28} \mathrm{Zu}$ den Assoziationsketten in Timms Roman und zu ihrer Rolle in der Struktur des Romans, s. Kilb apud Wiese 2016: 87.

${ }^{29}$ Romantisch ist ebenfalls das Lied, das Marga von Dahlem auf seine Bitte hin singt (HS 152), was als eine Variation des Motivs vom Gedichte Vortragen zu betrachten ist. Gleich in den ersten Seiten des Romans erwähnt Marga Eichendorff und Heine, als die Dichter, die sie in der Luft liest (HS 12).

${ }^{30}$ In HS beschreibt Marga das Erlebnis mit dem Regenbogen als ein außerordentliches Moment des Fliegens. Dabei wird das Erlebnis zwischen Cabo Juby und Fortaventura ["Aber durch das Gewölk hindurch schien strahlend die Sonne. So kam es, da $\beta$ ich in einem wundervollen, farbenprächtigen Regenbogen flog. Vom Flugzeug aus wirkt ein Regenbogen als geschlossener Kreis, der immer mitfolgt"

(KW 119)] auf ein Erlebnis über die Sierra de Guara (Spanien) transponiert: "Bei mir war es der Flug über Spanien gewesen [...] Ich flog in einen runden, einen in sich geschlossenen Regenbogen, und dieser Regenbogen mit seinen deutlichen Farbabstufungen flog - wie ein Wunder - mit mir mit” (HS 40).

31 Zum Motiv des Engels in HS, s. Bernsmeier 2014: 69-75. 